REVIEW

\title{
Recent Developments of the PHITS code
}

\author{
Koji NIITA $^{1^{*}}$, Hiroshi IWASE ${ }^{2}$, Tatsuhiko SATO ${ }^{3}$, Yosuke IWAMOTO ${ }^{3}$, \\ Norihiro MATSUDA ${ }^{3}$, Yukio SAKAMOTO ${ }^{3}$, Hiroshi NAKASHIMA ${ }^{3}$, Davide MANCUSI ${ }^{4}$ and Lembit SIHVER ${ }^{4,5}$ \\ ${ }^{1}$ Reaseach Organization for Information Science \& Technology (RIST), Naka, Ibaraki 319-1106, Japan \\ ${ }^{2}$ High Energy Accelerator Research Organization (KEK), Tsukuba, Ibaraki 305-0801, Japan \\ ${ }^{3}$ Japan Atomic Energy Agency (JAEA), Tokai, Naka, Ibaraki 319-1195, Japan \\ ${ }^{4}$ ChalmersUniversity of Technology, Goetheborg, Sweden \\ ${ }^{5}$ Roanoke College, VA, USA
}

\begin{abstract}
PHITS, a general-purpose Particle and Heavy Ion Transport code System, has been used for various research fields such as radiation science, accelerator and its shielding design, space research, medical application, material research, and so on. Further developments of the PHITS code by improving the reaction models, including new physical processes and providing better user interfaces, are actively performed by the collaboration between RIST, JAEA, KEK, and Chalmers University. Recent developments of the PHITS code are presented concerned with the functions for "neutron long beam line", high energy extension of the JQMD model, and event generator mode for low energy neutron transport.
\end{abstract}

KEYWORDS: PHITS, JAM, JQMD, Monte Carlo transport, event generator mode.

\section{Introduction}

Particle and heavy ion transport code is an essential implement for various research fields such as radiation science, accelerator and its shielding design, space research, medical application, material research, and so on. We have therefore developed a general-purpose particle and heavy ion transport Monte Carlo code system, PHITS ${ }^{1)}$. In the PHITS code, we have used two simulation codes $\mathrm{JAM}^{2)}$ (Jet AA Microscopic Transport Model) and JQMD ${ }^{3)}$ (JAERI Quantum Molecular Dynamics) for intermediate and high energy nuclear reactions. JAM is a simulation code based on INC (intra-nuclear cascade) model, which explicitly treats all established hadronic states including resonances with explicit spin and isospin as well as their anti-particles. On the other hand, JQMD is a simulation code based on the molecular dynamics. A typical feature of QMD compared with that of the INC model is that QMD can describe not only nucleon-nucleus reactions but also nucleus-nucleus reactions in the same framework. The JQMD code has been widely used to analyze various aspects of heavy ion reactions as well as of nucleon-induced reactions ${ }^{3,4)}$. By using these nuclear reaction models and evaluated nuclear data for low energy neutron reactions, PHITS has shown a great ability of carrying out the radiation transport analysis of various particles and heavy ions in a wide energy range. We are, however, still developing the PHITS code by improving the reaction models, including new physical processes and providing better user interfaces. In this paper, we report the present status and recent developments of the PHITS code.

\section{Overview of PHITS \\ 1. The Transport Particles and Energy Range.}

*Corresponding Author, E-mail:niita@ tokai.rist.or.jp

(C) Atomic Energy Society of Japan
In PHITS, neutrons can be transported from thermal energies up to $200 \mathrm{GeV}$. Below $20 \mathrm{MeV}$ down to $0.1 \mathrm{meV}$, neutrons are described in the same manner as in the MCNP4C code $^{5)}$ based on the Evaluated Nuclear Data such as the ENDF-B/VI ${ }^{6)}$, JENDL-3.3 ${ }^{7}$, and LA150 libraries ${ }^{8)}$. For the neutron induced ractions above $20 \mathrm{MeV}$, the simulation model JAM is used. For protons and other hadrons, JAM is also used above $1 \mathrm{MeV}$ up to $200 \mathrm{GeV}$. But for charged particles except for nuclei below $1 \mathrm{MeV}$, only the ionization process is considered until the charged particles are stopped. PHITS also uses Evaluated Nuclear Data for photon and electron transport in the same manner as in the MCNP4C code based on ITS version 3.0 code $^{9)}$. The energy range of electron and photon was restricted to the energy region $1 \mathrm{keV}-1 \mathrm{GeV}$, but the extension of the maximum energy of these particles has been done recently by including the EGS5 $\operatorname{code}^{10)}$.

One of the advantages of the PHITS code is that PHITS can transport nuclei in materials. Below $10 \mathrm{MeV} / \mathrm{n}$, only the ionization process for the nucleus transport is taken into account, but above $10 \mathrm{MeV} / \mathrm{n}$ the nucleus-nucleus collisions up to $100 \mathrm{GeV} / \mathrm{n}$ is described by the simulation code JQMD. The QMD simulation, as well as the JAM simulation, describes the dynamical stage of the reactions. At the end of the dynamical stage, excited nuclei are created and must be forced to decay in a statistical way to get the final observed state. In PHITS, the GEM model ${ }^{11)}$ (Generalized Evaporation Model) is employed for light particle evaporation and fission process of the excited residual nucleus.

\section{The Magnetic Field.}

For simulating the transport of charged particles and heavy ions, the knowledge of the magnetic field is sometimes necessary to estimate beam loss, heat deposition in the magnet, and beam spread. PHITS can provide dipole 
and quadrupole magnetic fields in any direction and any region of the setup geometry. In contrast to other beam transport codes, PHITS can simulate not only the trajectory of the charged particles in the field, but also the collisions and the ionization process at the same time. This is a great advantage of PHITS for the design study of the high intensity proton and heavy ion accelerator facilities, where one must estimate heat deposition, radiation damage of the magnets and the radiation shielding, as well as perform trajectory calculations.

\section{The Ionization Process.}

For the ionization process of the charged particles and nuclei, the SPAR code ${ }^{12)}$ is used for the average stopping power $\mathrm{dE} / \mathrm{dx}$, the first order of Moliere model for the angle straggling, and the Gaussian, Landau and Vavilov theories for the energy straggling around the average energy loss according to the charge density and the velocity of the charged particles. In addition to the SPAR code, the ATIMA package $^{13)}$ developed at GSI group has recently been implemented as an alternative code for the ionization process in PHITS.

\section{The Total and Elastic Cross Sections.}

The total reaction cross section, or the life time of the particle for decay is an essential quantity in the determination of the mean free path of the transport particle. According to the mean free path, next collision point is chosen using the Monte Carlo method. It is therefore very important that reliable data of total, non-elastic and elastic cross sections is used for the particle and heavy ion transport In PHITS, the Evaluated Nuclear Data is employed for neutron-induced reactions below $20 \mathrm{MeV}$. For neutron-induced reactions above $20 \mathrm{MeV}$, the parameterization is used ${ }^{14)}$. As for the elastic cross sections, the Evaluated Nuclear Data is also used for neutron-induced reactions below $20 \mathrm{MeV}$, and the parameterization is used above $20 \mathrm{MeV}^{14)}$. The parameterizations are also used for proton induced reactions for all energies, and for the double differential cross sections of elastic nucleon-nucleus reactions ${ }^{14)}$. Recently we have also adopted the NASA systematics developed by Tripathi et al., ${ }^{15}$ for the total nucleus-nucleus reaction cross section, as an alternative to the Shen formula ${ }^{16)}$.

\section{The Radioactivity Calculation.}

In order to calculate the induced radioactivity, we can use the burn-up code DCHAIN-SP2001 ${ }^{17)}$ incorporated with the PHITS code. By the PHITS calculations, one can obtain the production cross sections of nuclei produced by the high energy nuclear reactions and the neutron spectra below 20 $\mathrm{MeV}$ in 175 groups. These two outputs of the PHITS calculations are used as an input data of the burn-up calculation performed by the DCHAIN-SP code, in which 175 group neutron activation cross section data library, decay data library including $\gamma$-ray spectrum, and electron capture, $\beta^{+}$decay ratio data library are employed. As a result of the burn-up calculation by DCHAIN-SP, the number of produced nuclides, activity of nuclide, decay heat of nuclide and $\gamma$-ray spectrum are obtained as a function of time. One can also transport this $\gamma$-ray by using the PHITS code with the same geometry.

\section{Tally and Utilities.}

User interface and tally, which is a detector in the code to get desirable information from the simulation, are important equipment in a general-purpose transport code. PHITS provides a variety of tallies, which score track length, flux and current of crossing surface, produced particle and residual nucleus at the collision, average heat and distribution of deposit energy, star density, and DPA (displacements per atom). These quantities can be tallied in a cell defined by the geometry and also by the super-imposed $\mathrm{r}-\mathrm{z}$ and xyz scoring meshes. The meshes of energy, time and angel are additionally set for each tally quantities.

The final results of the tallies are written on a text file. At the same time, PHITS generates an EPS (Enhanced PostScript) file, which can be shown on the monitor as a graph, by the graphic utility ANGEL ${ }^{18)}$ included in PHITS. This function can be used even in running time of the calculation. So you can check immediately how the calculation proceeds and how the statistics of the tally quantities increases on your monitor during the calculation. This is a good utility in 3D Monte Carlo calculation, since we sometimes have to change the parameters of the variance reduction in an iterative way to get good statistics.

\section{Recent Developments of PHITS}

We have developed many new functions and tallies, improved reaction models, included new physical processes and provided better user interfaces in the PHITS code in these years. Here we picked up three topics of the recent developments of PHITS, (1) functions for neutron long beam line, (2) high energy extension of the JQMD code, and (3) event generator mode for low energy neutrons, and discuss the detail of these functions.

\section{Functions for Neutron Long Beam Line.}

For neutron scattering instruments at a 1-MW spallation neutron source facility in the Japan Proton Accelerator Complex (J-PARC), we have to study the performance of the neutron mechanical and optical devices with the beam-line shields in order to achieve the highest possible signal-to-background ratio. For this, we have developed the PHITS code further by including new functions for optical and mechanical devices for neutron scattering experiments such as supper mirror and T0 chopper, respectively. In addition to these functions for the optical and mechanical devices, PHITS has been also developed in order to simulate neutron transport under various external fields. PHITS supports the neutron transport simulations under gravity and under dipole, quadrupole and sectupole magnetic fields and user-defined magnetic fields by the finite element method for neutrons by introducing the Lamor precession of neutron spin as a dynamical variable. Furthermore, PHITS can simulate neutron transport and scattering process under a 
time dependent magnetic fields, which will be used to develop a neutron-focusing device for the small-angle neutron scattering instrument. In Fig. 1, we show a typical example of the neutron motion in the sextupole magnet with gravity field simulating the experimental setup at JRR-3 research reactor at JAEA. The strength of the magnet is $7.13 \times 10^{4} \mathrm{~T} / \mathrm{m} 2$, while the incident neutron is an unpolarized one with $13.4 \pm 3 \AA$ energy. One can see in this figure that the half is divergent and the other is focused according to the initial spin of the neutron affected by the gravity field.

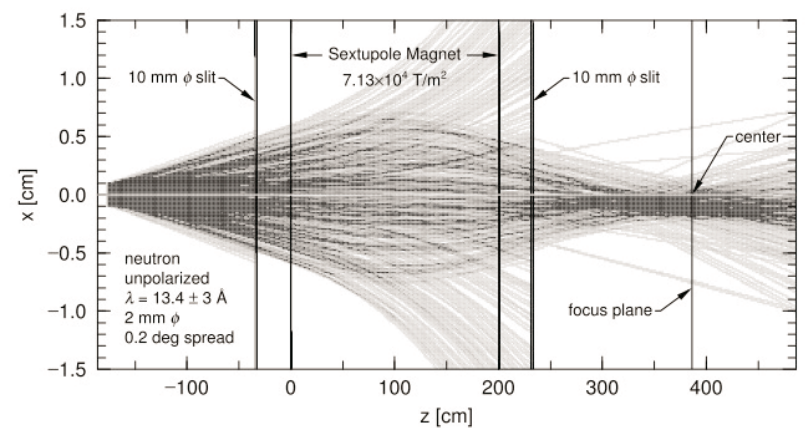

Fig. 1 Neutron tracks in the sextupole magnet field and gravity.

In contrast to the neutron ray-trace Monte Carlo simulation packages, PHITS can treat not only the neutron scattering processes for a ray tracing but also the transport processes for a shielding design by simulating neutron interactions with various materials and by production of secondary particles at the same time. This is a great advantage of PHITS for studying the beam-line shield design especially for "neutron long beam lines" in which the optical and mechanical devices play an important role for neutron scattering experiments. For an example, the neutron dose around the neutron beam-line after a curved guide tube is almost governed by the flux loss of thermal neutron at the surface of the supper mirror in the curved guide, since the high-energy ( $\mathrm{MeV}$ and $\mathrm{keV}$ level) neutrons are reduced drastically by the curved guide.

In addition to the equipments implemented in the PHITS code, one has to introduce special Monte Carlo techniques for the simulation of neutron transport through the long beam-line to obtain enough statistics in the final results. In fact, two-step calculations were introduced. In the first step, we calculate the neutron and photon flux at the entrance of each beam-line starting from a collision between target and incident protons. The calculated flux data for each beam-line are stored in a database and used as a source in the second step calculation for each beam-line from the moderator to the detectors. In the second step calculation for the beam-lines from the moderator to the detectors, we need another Monte Carlo technique for variance reduction, since the beam current transported through the beam-line decreases proportional to the inverse square of the distance from the moderator. This means that the current crossing the wall of the beam-line, which is called as "wall current", at $100 \mathrm{~m}$ point is six order of magnitude smaller than that at $1 \mathrm{~m}$ point from the moderator. To reduce this variance, we have introduced a special function of the particle source in which the wall current of the particle in the calculation is equalized at any point of the beam-line by changing the importance weight of the particle to simulate a real situation of the current inside the beam-line (we call this "duct source option"). The introduction of the Monte Carlo techniques to PHITS code highly reduces the computing time and provides a reliable calculation to design shielding for the long beam-line of the spallation neutron source facility at J-PARC.

To check the validity of this method, we have compared the results by this method with the results by one through calculation using the point estimator in MCNPX code. Figure 2 shows the results of the current inside the beam-line and the wall current as a function of the distance, $\mathrm{L}$, from the moderator. In an ideal case, the current is proportional to $1 / \mathrm{L}^{2}$, while the wall current is proportional to $1 / \mathrm{L}^{3}$. The deviation of the calculation results compared to the ideal case around a small $\mathrm{L}$ region is due to the beam flux coming into the beam-line from the margin area of the moderator to the size of the cross-section of beam guide. The wall current calculated with MCNPX is derived by the derivative of the current. Therefore there exist some fluctuations in the wall current by the MCNPX calculation, though the current is nearly smooth. On the other hand, the duct source calculated with PHITS shows a good agreement with that calculated with MCNPX and is very smooth as wall current.

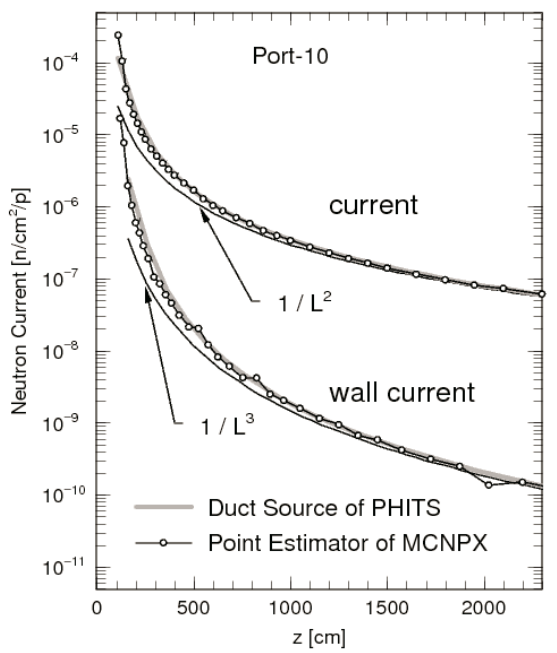

Fig. 2 Comparison of the current and wall current calculated by the duct source of PHITS with the results by the point estimator in MCNPX.

\section{High Energy Extension of the JQMD code}

The JQMD model was developed with the intent to provide a unified description of various aspects of nuclear reactions. By combining JQMD and the statistical decay model GEM, one obtains a hybrid model that can describe accurately both the fast dynamical stage and the slow statistical stage of the reaction and, thus, reproduce measured double-differential cross sections for the production of protons and neutrons in proton-nucleus ${ }^{3,4)}$ and nucleus-nucleus collisions ${ }^{19)}$. There are other observables, however, that JQMD does not reproduce as accurately as double-differential cross sections for nucleon production; for 
example, fragment yields in heavy-ion reactions are sometimes in sensible discrepancy with the experimental data ${ }^{20)}$, especially for soft, peripheral reactions that only entail stripping of a small number of nucleons. The cause of these shortcomings can be traced back to a small intrinsic instability of the ground state of the JQMD nucleus, which in fact can emit nucleons spontaneously because of potential-energy fluctuations and alter significantly the final yields of soft, peripheral reactions (hard, central collisions are not very sensitive to the details of the initial configuration).
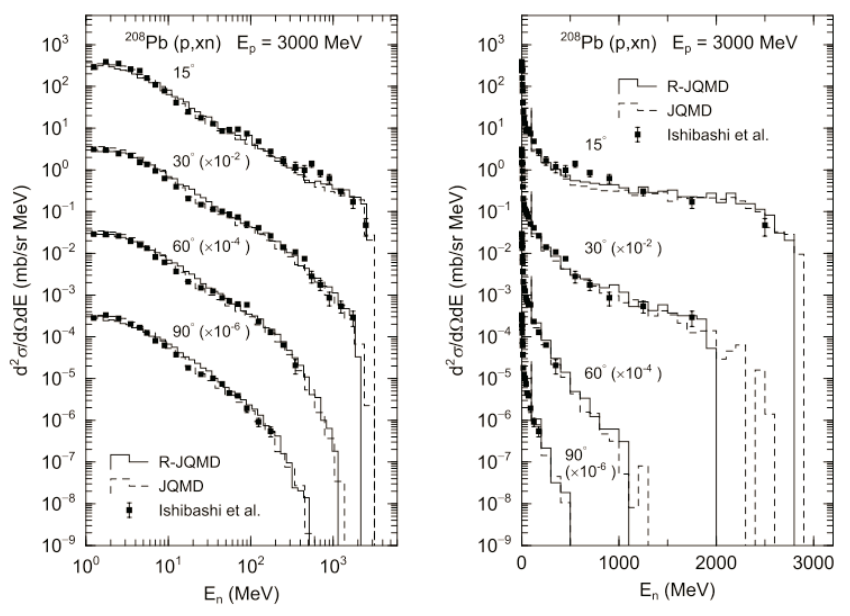

Fig. 3 Double-differential energy-angle cross sections for the production of neutrons in the reaction between $3-\mathrm{GeV}$ protons and lead, with data taken from Ref.22. Left: log scale; right: linear scale. No significant difference can be observed between JQMD and R-JQMD.

These instabilities have two main reasons: firstly, the JQMD formalism is not completely relativistically covariant, which implies that the dynamics of a nucleus is somehow dependent on the frame of reference used. Secondly, the ground state that JQMD nuclei are initialized in is only an approximation; in all QMD models, it is conceptually impossible to create the "real" ground state, i.e. the lowest-energy eigenstate of the quantum-mechanical n-body Hamiltonian operator, which is influenced by Pauli's exclusion principle. (In a semi-classical n-body theory, like QMD-models, Pauli's principle is not taken into account and particles are thus allowed to move closer to each other). If we want to describe peripheral nucleus-nucleus reactions consistently in the JQMD framework, it is necessary to switch to a relativistically covariant formalism and to improve the ground-state initialization algorithm, in order to suppress spurious potential-energy fluctuations and particle decays. Recently, Mancusi et al. has thus introduced R-JQMD ${ }^{21)}$, an improved and fully covariant version of JQMD that also features a new ground-state initialization algorithm for nuclei. This new version of JQMD will be included in the PHITS code in the near future, and expected to give much reliable results for the fragmentation cross sections at high energy heavy-ion reactions. The double differential cross sections of neutron from proton induced reactions, for an example, are not as sensitive to relativistic effects, thus, one should not expect large differences in the results by R-JQMD and JQMD. We have verified this by computing double differential energy angle cross sections in Fig. 3. It is clear that R-JQMD and JQMD do not differ very much as far as neutrons spectra are concerned ${ }^{21)}$.

\section{Event generator Mode for Low Energy Neutron.}

Most of the Monte Carlo transport calculations are based on Boltzmann equation for one-body phase space distribution of the transport particles. In such transport calculations, one can obtain only the mean value of the one-body observables in the phase space, e.g. heat, flux, and dose, but one cannot calculate the fluctuations around the mean value. Recently, however, the higher order quantities, i.e. the fluctuations around the mean values of the one-body observables are often required. A typical example for such a correlated quantity is the deposit energy distribution in a cell, which is necessary to estimate the response function of detectors, the single event upset probability of semiconductor memory cells and the radiation effects in a micro-dosimetric treatment. The solution of the Boltzmann equation cannot describe the distribution but only the mean value. Furthermore, Monte Carlo calculations by using the nuclear data cannot deal with these quantities, since the nuclear data includes only the inclusive one-body cross sections but no information of the correlations. We have therefore developed a new treatment of radiation behaviour for the transport of the low energy neutrons by introducing an event generator model, in which we have combined the nuclear data and a special reaction model so as to trace all higher correlations among ejectiles keeping the energy and momentum conservation in a collision.

For high energy transport calculations, the nuclear reaction models such as JAM and JQMD are commonly used instead of the evaluated nuclear data to describe the ejectiles from each nuclear reaction. The reaction models usually generate each event with keeping the energy and momentum by the Monte Carlo method. In this sense, the reaction model is called as "Event Generator". Therefore we can extract any information from the transport calculations with such reaction models. On the other hand, the transport calculations based on the Boltzmann equation with the nuclear data have no such concept of "Event". For the actual numerical solution of the Boltzmann equation, one usually employs the test particle method where the one-body phase-space distribution function is evaluated by integrating the test particle tracks in the phase-space. In this numerical calculation, things are going on in a very similar manner as in the high energy transport calculation with the event generator. However, there is no "Event" in this type of calculations. One history in this type of calculation has no physical meaning but gives us only a statistical weight. The observables are obtained after averaged out the statistical weight. There is no physical meaning in the distribution around the mean value but only the statistical variance. However, the distribution around the mean value calculated in the high energy transport code with the event generator mode is a real physical quantity, which is often required as mentioned above. Therefore, we have developed a model to determine the energy and momentum of all ejectile in event 
by event by using the information of the nuclear data and combining a special statistical decay model, which is described in the followings.

At first, we use the total cross section and the channel cross sections from the evaluated nuclear data to choose a channel of the collision. We categorize the channels by the number of outgoing neutrons. For the channel which does not produce neutrons, we call this "capture" channel, the excitation energy and momentum of the captured nucleus is determined uniquely from the incident energy of neutron and target nucleus. For the decay process of this excited nucleus, we apply a special statistical decay model, in which the decay width of neutron is assumed to be zero for the capture channel. Considering the charged particle decay and photon decay in a statistical way, we can determine all energy and momentum of ejectiles and residual nucleus for the capture channel.

For the elastic channel, which is one of the channels categorized as one outgoing neutron, we choose the scattering angle of outgoing neutron by the Monte Carlo method according to the evaluated nuclear data. We can then determine the energy of neutron and the momentum of the recoil nucleus uniquely by the kinematics of the elastic collision.

Next channel is the inelastic channel of (n,n') case, which is also a channel with one outgoing neutron. For this case, we first choose the momentum of the outgoing neutron according to the double differential cross sections of the evaluated nuclear data by Monte Carlo method. By the kinematics of this emission, we can uniquely determine the excitation energy and the momentum of the residual nucleus. We then apply the same statistical decay procedure as in the capture channel without neutron decay width since there is no more neutron emission after the first neutron emission for this channel.

Finally for (n,Nn') channel, which is a channel with $\mathrm{N}$ outgoing neutrons, we choose the momentum of the first outgoing neutron in the same way as in the (n,n') case. After one nucleon emission, we apply only neutron decay procedure to the excited nucleus until $\mathrm{N}$ nucleons are emitted. After $\mathrm{N}$ neutrons are emitted, the special decay procedure without neutron decay width is applied.

By the above procedure, we can treat a low energy neutron collision as an "Event", keeping the energy and momentum conservation and preserving the channel cross sections and the inclusive neutron double differential cross sections, which are given by the evaluated nuclear data.

By the event generator mode in PHITS for low energy neutron transport phenomena, we can calculate many new quantities which cannot be obtained by MCNP-type calculation, e.g. kinetic energy distribution of residual nuclei and charged particles, two-particle correlation, and so on. Here we show an example of the event generator mode in PHITS, i.e. deposit energy distribution in a cell, which is a typical quantity beyond one-body observable. This quantity is well known as a pulse height tally in MCNP. However, this is a conceptually wrong quantity to be treated in the MCNP-type calculation as discussed before. As mentioned in the MCNP manual, this tally is very restricted to use for neutron transport in MCNP. Namely, this tally does not work if a collision produces multiple neutrons.

Figure 4 shows the deposit energy distribution in a thin $\mathrm{Si}$ tip irradiated with mono energetic neutron at $19 \mathrm{MeV}$. The thickness of the Si tip is $3 \mu \mathrm{m}$. If we calculate the deposit energy by using the Kerma factor in MCNP-type calculation, we get a delta-function-like single peak at $100 \mathrm{eV}$ with almost no distribution. However, Fig.4 shows broad distribution obtained by the event generator mode in PHITS, where the deposit energy is estimated from the ionization losses of the charged particles and the recoil nuclei. This distribution is not due to a statistical fluctuation in the Monte Carlo numerical calculation but a physical quantity to be considered. This distribution is also very important to estimate the single event upset probability of semiconductor memory cells, since only the events which induce the deposit energy greater than a certain threshold energy contribute to the error.

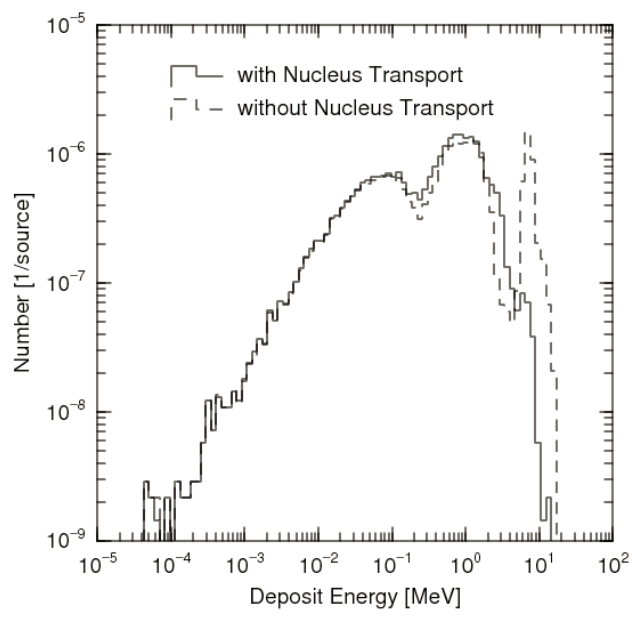

Fig. 4 Deposit energy distribution in a thin $(3 \mu \mathrm{m})$ Si tip irradiated by mono energetic neutron at $19 \mathrm{MeV}$. The solid line is the result of the event generator mode of PHITS with nucleus transport, and the dashed line is the result by a local approximation, i.e. without nucleus transport.

In Fig. 4, we have plotted two distributions, solid line and dashed line. The dashed line was obtained by the local approximation, in which all charged particles and recoil nuclei produced in neutron induced reactions are assumed to be stopped and deposit their kinetic energy at the collision point. This approximation is valid under the condition where the range of charged particles is much smaller than the size of the system. For this example, the both are comparable, since the range of charged particles is of the order of $\mu \mathrm{m}$, while the thickness of $\mathrm{Si}$ is $3 \mu \mathrm{m}$. Therefore we transported the charged particles and the recoil nuclei in the PHITS calculation without the local approximation. The result is shown by the solid line in Fig. 4. This shows that the highest peak of the dashed line is disappeared. This means that some part of the charged particles or the recoil nuclei (mainly alpha particles for this case) can escape from $\mathrm{Si}$ tip to the outside.

This example indicates two important points. The first is that the deposit energy distribution in a cell is well described by the event generator mode in PHITS even for low energy 
neutron transport below $20 \mathrm{MeV}$. The second is that the local approximation is not valid for the system whose size is comparable to the range of the transport charged particles and recoil nuclei.

\section{Conclusion}

We have developed the general-purpose Particle and Heavy Ion Transport code System PHITS, which is an essential implement for various research fields such as radiation science, accelerator and its shielding design, space research, medical application, material research, and so on. Description of nuclear reactions in the code is one of the key quantities to determine the accuracy of the transport code. In PHITS, we have incorporated two important models, JAM and JQMD, to simulate the particle induced reactions up to $200 \mathrm{GeV}$ and the nucleus-nucleus collisions, respectively.

In this report, we have briefly presented the models incorporated in the PHITS code and discussed the present status and summarized the recent developments of the code. We have picked up three topics of the recent developments of PHITS, (1) functions for neutron long beam line, (2) high energy extension of the JQMD code, and (3) event generator mode for low energy neutrons, and discuss the detail of these functions. By these new functions, PHITS has had a much wider ability of carrying out the radiation transport analysis of almost all accelerator facilities of protons and heavy ions within a wide energy range, as well as the possibilities to be used in a wide fields of applications, e.g. semiconductor soft errors, BNCT, radiotherapy, and radioprotection of personnel on space missions.

\section{References}

1) H. Iwase, K. Niita and T. Nakamura, J. Nucl. Sci. Technol. 39, 1142 (2002); K. Niita, et al., "Development of General-Purpose Particle and Heavy Ion Transport Monte Carlo Code", Radiat. Meas. 41, 1080 (2006).

2) Y. Nara, N. Otuka, A. Ohnishi, K. Niita and S. Chiba, "Relativistic nuclear collisions at $10 \mathrm{AGeV}$ energies from $\mathrm{p}+\mathrm{Be}$ to $\mathrm{Au}+\mathrm{Au}$ with the hadronic cascade model", Phys, Rev. C61, 024901 (2000).

3) K. Niita, et al., "Analysis of the $(\mathrm{N}, \mathrm{xN}$ ') Reactions by Quantum Molecular Dynamics Plus Statistical decay Model”, Phys. Rev. C52, 2620 (1995).

4) S. Chiba, O. Iwamoto, T. Fukahori, K. Niita, Toshiki Maruyama, Tomoyuki Maruyama, A. Iwamoto, "Analysis of proton-induced fragment production cross sections by the quantum molecular dynamics plus statistical decay model”, Phys. Rev., C54, 285 (1996), S. Chiba, M.B. Chadwick, K. Niita, Toshiki. Maruyama, Tomoyuki. Maruyama, A. Iwamoto, "Study of the Nucleon-Induced Preequilibrium Reactions in Terms of the Quantum Molecular Dynamics”, Phys. Rev., C53, 1824 (1996).

5) J.F. Briesmeister, Editor. "MCNP - A General Monte Carlo N-Particle Transport Code, Version 4C", LA-13709-M (2000).

6) V. McLane, et al., "ENDF/B-VI Summary Documentation", BNL-NCS-17541 (1996).
7) K. Shibata, et al., "Japanese Evaluated Nuclear Data Library Version 3 Revision-3: JENDLE-3.3”, J. Nucl. Sci. Technol. 39, 1125 (2002).

8) M. B. Chadwick, et al., "LA150 Documentation of Cross Sections, Heating, and Damage", Los Alamos National Laboratory report; LA-UR-99-1222 (1999).

9) J. A. Halbleib, et al., "ITS Version 3.0: The Integrated TIGER Series of Coupled Electron/Photon Monte Carlo Transport Codes", SAND91-1634 (1992).

10) H. Hirayama, et al., "The EGS5 Code System", Report SLAC-R-730, Stanford Linear Accelerator Center, Stanford, CA, (2005).

11) S. Furihata, "Statistical analysis of light fragment production from medium energy proton-induced reactions", Nucl. Instr. and Meth. B171, 251 (2000).

12) T. W. Armstrong and K. C. Chandler, "A Fortran program for computing stopping powers and ranges for muons, charged pions, protons, and heavy ions". ORNL-4869, Oak Ridge National Laboratory, (1973).

13) C. Scheidenberger and H. Geissel, ,Slowing down of relativistic heavy ions and new applications", Nucl. Instr. Meth. B136, 114 (1998),

14) K. Niita, H. Takada, S. Meigo and Y. Ikeda, "High-energy particle transport code NMTC/JAM", Nucl. Instr. and Meth. B184, 406 (2001).

15) R. K. Tripathi, F. A. Cucinotta, and J. W. Wilson, "Accurate universal parametrization of absorption cross sections", Nucl. Instr. and Meth. B117, 347 (1996), R. K. Tripathi, F. A. Cucinotta, and J. W. Wilson, "Accurate universal parametrization of absorption cross sections III - light systems", Nucl. Instr. and Meth. B155, 349 (1999).

16) W. Shen, B. Wang, J. Feng, W. Zhan, Y. Zhu and E. Feng, "Total reaction cross section for heavy-ion collisions and its relation to the neutron excess degree of freedom", Nucl. Phys., A491, 130 (1989).

17) T. Kai et al., "DCHAIN-SP2001: High Energy Particle Induced Radioactivity Calculation Code", JAERI-DATA/CODE, 2001-016 (in Japanese) February 2001.

18) K. Niita, unpublished.

19) Y. Iwata, et al., "Double-differential cross sections for the neutron production from heavy-ion reactions at energies E/A=290-600 MeV", Phys. Rev. C64, 054609 (2001), T. Kurosawa, et al., "Spectral Measurements of Neutrons, Protons, Deuterons and Tritons Produced by $100 \mathrm{MeV} /$ nucleon $\mathrm{He}$ Bombardment", Nucl. Instr. and Meth. A430, 400 (1999), "Neutron yields from thick $\mathrm{C}, \mathrm{Al}, \mathrm{Cu}$, and $\mathrm{Pb}$ targets bombarded by $400 \mathrm{MeV} /$ nucleon $\mathrm{Ar}, \mathrm{Fe}$, Xe and $800 \mathrm{MeV} /$ nucleon Si ions", Phys. Rev. C62, 044615 (2000).

20) L. Sihver, et al., "Recent developments and benchmarking of the PHITS code", Adv. Space Res. 40, 1320 ( 2007).

21) Davide Mancusi, Koji Niita, Tomoyuki Maruyama, Lembit Sihver, "Stability of nuclei in peripheral collisions in the JAERI quantum molecular dynamics model”, Phys. Rev. C79, 014614 (2009).

22) Ishibashi, et al., "Measurement of Neutron-Production Double-Differential Cross Sections for Nuclear Spallation Reaction Induced by $0.8,1.5$ and $3.0 \mathrm{GeV}$ Protons", J. Nucl. Sci. Technol. 34, 529 (1997) 529. 